\title{
Can Nanofluids Lead to Commercial Usage in Solar Engineering
}

Khalaji Assadi $\mathbf{M}^{*}$ and Reza Shamshirgaran S

Department of Mechanical Engineering, University Technology Petronas, Malaysia

*Corresponding author: Khalaji Assadi M, Department of Mechanical Engineering, University Technology Petronas, Malaysia, Tel: +6053687202; E-mail: morteza.assadi@petronas.com.my

Received date: March 07, 2016, Accepted date: March 10, 2016, Published date: March 12, 2016

Copyright: @ 2016 Assadi MK, et al. This is an open-access article distributed under the terms of the Creative Commons Attribution License, which permits unrestricted use, distribution, and reproduction in any medium, provided the original author and source are credited.

\section{Nanofluids in Solar Engineering}

The efficiency concept has been the major concern of specialists and analysts in the vital flied of energy. The energy efficiency corresponds with the productivity of resources consumption since there is a worrying limitation on the fossil fuel reserves of the earth. Although the price of fuel has been diminished significantly in recent years, the constraint of its availability in not-too-far future has put the pressure on the governments to intend toward the renewable energies more than ever. Furthermore, nobody can really estimate that what would be occurred in the global economic market in the next years.

Solar energy considered as one of the most reliable and available sources of energy which can be renewed continuously. That is why the different technologies, i.e. PV and thermal systems, have been developed and studied yet. Apart from the improvements which originated from design and technology progress, the appearance of nanotechnology caused significant advancement in solar engineering similar with the other fields of study. There are numerous researches on the field of solar energy with the applications of nanotechnology. For instance, currently the solar energy can be observed in heating, cooling, power generation, energy storage, transportation, medical etc. As long as nanotechnology proposed, the solar engineers has made their efforts to enhance the performance of solar systems and achieve the higher efficiencies. In the case of solar collector systems, nanomaterial's can be added into absorber surfaces or heat transfer fluids which would result in enhancement of thermal and optical properties of the systems. Nanomaterial's categorized as follows:

Organic: fullerene, nanotube, electrospun nanofibers Inorganic: metal, metal oxides, quantum dots hybrid.

Based on the application, nanomaterial's can be mixed with the fluids which called Nanofluids. In other words, the suspension of nanoparticles in liquids considered as Nanofluids. Common liquids as the base fluid are water, ethylene, glycol and oil and the nanoparticles which have been used in literatures for dispersing in base fluid include carbon nanotube, alumina, titanium dioxide, silver, copper, graphite, etc. Nanofluids are made by mechanical (one-step or two-step) or chemical methods [1]. The synthesis is the main process which involved in preparation of the Nanofluids. On the other hand, the combination of nanomaterial's with phase change materials (PCMs) known as Nano composites. Each type of Nanofluids or Nano composites exhibits the special properties. For example, the Nanofluids are more applicable in solar collectors for power generation and Nano composites are preferably considered in thermal storage projects i.e. heat or cold storage which would provide the industrial or domestic requirements of different climates. The hybrid systems exploit the most benefits of nanomaterials for simultaneous power generation and thermal storage. Furthermore, using the PCMs can increase the operating time of the system during the night or cloudy situations.

Nanofluid phase change materials (NPCMs) have opened a new field of study for researchers. In fact, a NPCM is a liquid in which nanoparticles that change phase are added in order to enhance the thermal properties of this fluid.

Despite the fact that all researches have been conducted theoretically and experimentally in all around the world, prove that the application of Nanofluids in solar engineering would lead to enhancement of optical and thermo-physical (conductive and convective) behaviour of solar systems, the applications of Nanofluids limited to the noncommercial projects [2]. Two main reasons interfere with the aim of commercializing Nanofluids application for solar power generation or solar thermal storage. Firstly, the results of remarkable achievements of numerous researchers show an inconsistency in some cases and therefore, the uncertainty of the results may necessitate more detail studies to overcome some ambiguities. Secondly, it is obvious that the optical and thermal properties of Nanofluids would be enhanced rather the base fluids, however, the issues of their application including durability, sedimentation, agglomeration, viscosity problems, some design complexities and operating costs have not been studied exactly and sufficiently. Hence it seems there would be a rather long way to achieve the exact, clear and unique results to smooth commercializing of Nanofluids applications in solar engineering.

\section{References}

1. Leong KY, Chyuan OH, Amer NH, Norazrina MJ, Risby MS, et al. (2016) An Overview on Current Application of Nanofluids in Solar Thermal Collector and Its Challenges. J Renewable and Sustainable Energy Reviews 53: 1092-1105.

2. Javadi FS, Rahman S, Kamalisarvestani M (2013) Investigating Performance Improvement of Solar Collectors. J Renewable and Sustainable Energy Reviews 28: 232-245. 